(c) The Author(s) 2016. This is an Open Access article, distributed under the terms of the Creative Commons Attribution licence (http://creativecommons. $\mathrm{org} /$ licenses/by/4.0/), which permits unrestricted re-use, distribution, and reproduction in any medium, provided the original work is properly cited.

\title{
Glacier shrinkage across High Mountain Asia
}

\author{
J. Graham COGLEY \\ Department of Geography, Trent University, Peterborough, ON, Canada \\ Correspondence: J. Graham Cogley<gcogley@trentu.ca>
}

\begin{abstract}
An assessment of glacier shrinkage (reduction of area) for all of High Mountain Asia requires a complete compilation of measured rates of change and also a methodology for objective comparison of rates. I present a compilation from 155 publications reporting glacier area changes, and also a methodology that overcomes the main obstacles hindering comparison. Glacier areas are not always assigned uncertainties, and this problem is addressed with an error model derived from published estimates. The problem of discordant survey dates is addressed by interpolating measured areas to fixed dates at pentadal intervals. Interpolation error depends only incoherently on the time span between measurements, but strongly on glacier size: smaller glaciers, in addition to changing more rapidly on average, exhibit more variable rates of change. The overlapping boundaries of study regions are reconciled by mapping all of the information to a $0.5^{\circ}$ geographical grid. When coupled with glacier area information from the Randolph Glacier Inventory, the widely observed inverse dependence of shrinkage rates on glacier size shows promise as a tool for treating incomplete spatial coverage. Over High Mountain Asia as a whole from 1960 to 2010 , the unweighted average shrinkage rate is $-0.57 \% \mathrm{a}^{-1}$, but corrections for variable glacier size raise the average to $-0.34 \% \mathrm{a}^{-1}$, and filling unmeasured gridcells with rates based on size dependence alters the latter estimate to $-0.40 \% \mathrm{a}^{-1}$. The uncertainties in these rates are large. The Karakoram anomaly is found to be a zonal feature extending well to the east of the Karakoram proper.
\end{abstract}

KEYWORDS: glacier cover, glacier fluctuations, glacier mapping

\section{INTRODUCTION}

Glacier shrinkage across High Mountain Asia is documented in a voluminous and fast-growing literature that is analyzed here with two related aims: to develop methods for treating large numbers of area-change measurements jointly in an objective way; and to make an exploratory assessment of region-wide rates of shrinkage and possibly of changes in those rates.

The second aim cannot be realized until the first has been tackled, and the first cannot be tackled without a systematic and preferably complete compilation of the published information. There are practical difficulties at each of these steps. The literature is diffuse, and few or no authors have the time to submit their area-change measurements to the databases of the World Glacier Monitoring Service and the Global Land Ice Measurements from Space initiative. The starting and ending dates of the measurements are distributed haphazardly because of the need to avoid clouds and seasonal snow in source imagery, and spatial coverage varies according to the needs of each particular study. Each regional study has value for its own region, and may offer important insights into the response of glaciers to climatic forcing, but if the literature is to become greater than the sum of its parts it seems essential to interpolate the measurements to a regular space-time grid. A convenient grid is available as part of the Randolph Glacier Inventory (Arendt and others, 2014; Pfeffer and others, 2014).

The Randolph ice cover is particularly extensive in an arc from the Hengduan Shan in the southeast to the Pamir in the northwest, and further north in the central Tien Shan (Fig. 1a). The geographical pattern exhibited by mean glacier size (Fig. 1b) is different. Mean sizes less than $1 \mathrm{~km}^{2}$ are common across the region. However, in the Pamir, Karakoram, Kun Lun, central Tibet and the Hengduan Shan, larger glaciers, although few, increase the mean size considerably. The mean size in the eastern Himalaya is also seen in Figure $1 \mathrm{~b}$ to be moderately larger than the regionwide norm. The geography of both ice coverage and glacier size will be shown below to be relevant for interpreting the geography of shrinkage rates.

Methods for the treatment and comparison of area-change measurements are explained in the next section. The resulting uncertainties require detailed consideration, and this is the subject of Section 3. Section 4 presents exploratory results, and Section 5 offers a concluding discussion.

\section{COMPILATION AND PROCESSING OF MEASUREMENTS}

Series of two or more dated areas of 'objects' (either single glaciers or glacierized regions), with date ranges when necessary and area uncertainties when given in the source, were collected from the literature. In all, 155 sources of information about High Mountain Asia were identified. They are listed in the Supplementary Information (http://igsoc.org/ hyperlink/71a040_supp.pdf). All series that passed checks for measurement quality (e.g. freedom from arithmetic error) and for completeness of information were accepted. This means that for several objects multiple series were available. Such multiples were averaged during later processing. That is, they were treated as replicate measurements of the same physical quantity. They may be based on, for example, different measurement methods or different definitions of the word 'glacier', but it is assumed here that results are consistent within individual studies, and thus that replicate shrinkage rates are likely to be directly comparable with one another.

Auxiliary information about the number of glaciers measured and the completeness of regional measurements (whether covering all glaciers or just a sample) was attached to each area. Representative points or outlines for each 

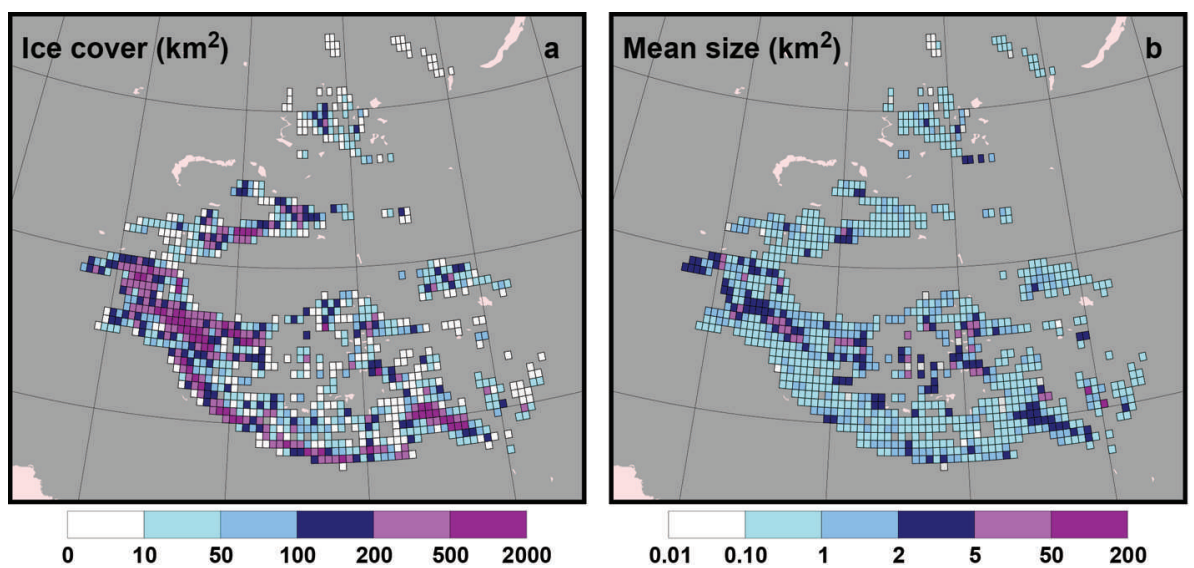

Fig. 1. Glacierization of High Mountain Asia from the $0.5^{\circ}$ grid of version 4.0 of the Randolph Glacier Inventory. (a) Ice coverage; total cell area decreases from $2796 \mathrm{~km}^{2}$ at $25^{\circ} \mathrm{N}$ to $1762 \mathrm{~km}^{2}$ at $55^{\circ} \mathrm{N}$, and the most heavily glacierized cell has $60 \%$ ice cover. (b) Mean glacier area. Albers equal-area projection of the World Geodetic System 1984 (WGS84) ellipsoid, with standard parallels at $32^{\circ} \mathrm{N}$ and $48^{\circ} \mathrm{N}$.

object, taken from maps in the relevant source, were digitized on-screen with the Global Land One-Km Base Elevation (GLOBE) digital elevation model as topographic background (Hastings and Dunbar, 1999).

Measurement dates, initially tabulated in the form YYYYMMDD, were converted to decimal years. When the date was incomplete, MMDD was set to 0701 or $D D$ to 15 , that is, to the middle of the implied time span. When only a range of dates was available, most commonly because of unavoidable use of multiple images, the middle of the date range was assigned to the measurement. The date range itself was the basis for calculating uncertainty in the date (Section 3).

Shrinkage rates were calculated as

$$
\dot{S}=\frac{1}{S_{0}} \frac{\Delta S}{\Delta t}=\frac{1}{S_{0}} \frac{S_{1}-S_{0}}{t_{1}-t_{0}}
$$

where $S\left(\mathrm{~km}^{2}\right)$ denotes areas and $t$ denotes dates, subscript 0 representing the earlier and subscript 1 the later of any two measurements of the area of any object. Division by the initial area $S_{0}$ converts rates in $\mathrm{km}^{2} \mathrm{a}^{-1}$ to fractional rates, which is essential if rates from different objects are to be compared. This is a 'simple-interest' calculation, not a 'compound-interest' calculation (Zemp and others, 2014).

The data, both areas and rates, of all time series were interpolated to pentadal dates separated from the reference date 1960.5 by integer multiples of 5 years. The start and end of each series were extrapolated backwards or forwards by up to 2 years. That is, each term in the pentadal series was required to be based on at least 3 years of coverage by an area-change measurement. Pentadal shrinkage rates were recalculated as in Eqn (1) from the averaged, interpolated series of pentadal areas.

Pentadal series from single glaciers were copied to the $0.5^{\circ}$ cell to which the glacier belongs. Series from glacierized regions were copied to each glacierized $0.5^{\circ}$ cell within their corresponding outlines (so that many pentadal rates appear in several contiguous gridcells). Within each $0.5^{\circ}$ cell, all series of single-glacier fractional rates were summed and averaged without weighting, and the result was summed and averaged together with all of the series of glacierized-region rates, again without weighting.

In all, 674 time series of shrinkage rates were obtained from 574 distinct objects, of which 248 were single glaciers and 326 were glacierized regions. The number of area measurements in the collection of time series was 1955 . The longest series contained 33 areas, but the average number of areas per series was 2.9. The oldest area measurement dates to the late 17th century, but for present purposes all pentads before 1960-65 were neglected. Of the 934 glacierized $0.5^{\circ}$ cells in High Mountain Asia, 630 had pentadal time series terminating after 1960 (Table 1). Few series end after 2005, and none after 2010. The number that begin before 1960 (e.g. all 71 of those in the $>50$ column) is considerable.

Because many sources omitted, or presented only generalized estimates of, area uncertainty, the area errors adopted here were assumed to depend only on the area itself, as in Pfeffer and others (2014). For this purpose (explained in detail in Section 3) a large sample is desirable, and published estimates of uncertainty are therefore drawn from a larger dataset of worldwide scope. They number 540 in total and are taken from 55 sources (Supplementary Information (http://igsoc.org/hyperlink/71a040_supp.pdf)). To estimate errors due to interpolation in time, series of three or more area measurements are necessary, and these too are taken from multiple sources worldwide.

To investigate methods for extrapolating measured shrinkage rates to unmeasured regions, six Asian 'multisets' were obtained. A multiset is a collection of tens to hundreds of dated shrinkage rates, with initial glacier areas, digitized from a published graph or table. Except that they lie within a study region of known extent, the locations of the individual glaciers are not known. The multisets contributed 1669 shrinkage rates, with start dates between 1957 and 1972 and end dates between 2003 and 2006. Thus in pentadal terms they all covered the period 1970-2005. A further 34 tabulated distributions of size-dependent shrinkage rate were obtained from 29 sources (Supplementary Information (http://igsoc.org/hyperlink/71a040_supp.pdf)). These distributions contained from four to eight rates (183 in all) averaged within logarithmic area classes.

\section{ESTIMATION OF ERRORS}

\subsection{Measurement uncertainty and temporal uncertainty}

Selected explicit and careful error estimates from the literature were grouped into logarithmic area classes and sorted within each class. In order to represent the 
size-dependent error $\mathrm{e}_{s}(S)\left(\% \mathrm{a}^{-1}\right)$, the power law

$$
e_{s}=e_{s 1} S^{\sigma}
$$

was fitted to the 68th percentiles of the frequency distributions of each of 12 area classes (Fig. 2a). The bestfitting parameters were $e_{s}=10.7$ and $\sigma=-0.184$. $e_{s}$ is the percentage uncertainty in a measured area of $1 \mathrm{~km}^{2}$. Although it does not address the striking long tails of the distributions, the choice of the 68th percentile to represent the observed $e_{s}(S)$ is an improvement over the arbitrary correction factor relied upon by Pfeffer and others (2014). To place all of the information on a common footing, Eqn (2) was used to calculate errors for all areas in the dataset from both single glaciers and glacierized regions, whether or not they were accompanied by their own errors.

Equation (2) was also used to calculate errors for all pentadal interpolated areas. For these it is also necessary to estimate the error due to interpolation. This error is likely to depend on the total time span of the area-change measurement and possibly on the times between the interpolation date and each endpoint of the area-change measurement. Graphs of these dependencies (not shown) exhibit strong variability but no coherent behaviour. In contrast, the error due to interpolation depends quite coherently on the initial area of the object, as in Figure $2 b$, where the quantity on the vertical axis is

$$
\delta S_{j}= \begin{cases}\widehat{S}_{j} / S_{j}-1 & \text { if } \widehat{S}_{j}>S_{j} \\ S_{j} / \widehat{S}_{j}-1 & \text { if } \widehat{S}_{j} \leq S_{j}\end{cases}
$$

Here $j$ is the index of a date $t_{j}$ at which there is a measurement $S_{j}$, and $t_{j}$ lies between two other dates $t_{i}$ and $t_{k}$ at which there are also area measurements. $\widehat{S}_{j}$ is the area obtained at date $t_{j}$ by linear interpolation between $t_{i}$ and $t_{k}$. In Eqn (3) we transpose numerator and denominator when needed to ensure that $\delta S_{j}$ is non-negative, because when evaluating the interpolation error there is no reason to distinguish between time series of $S$ that are concave and convex.

To put Eqn (3) to use we scan the entire global dataset of area-change measurements to identify time series of length $n \geq 3$, and interpolate at the dates of all intermediate measurements in the nested sub-series $\{1, \ldots, n$; $2, \ldots, n-1 ; \ldots\}$, requiring each sub-series to be at least 0.5 year long. These sub-series all have different start and end dates.

The resulting 7320 estimates of $\delta S_{j}$ are then grouped and sorted just as in Figure $2 \mathrm{a}$, and a similar power law is fitted

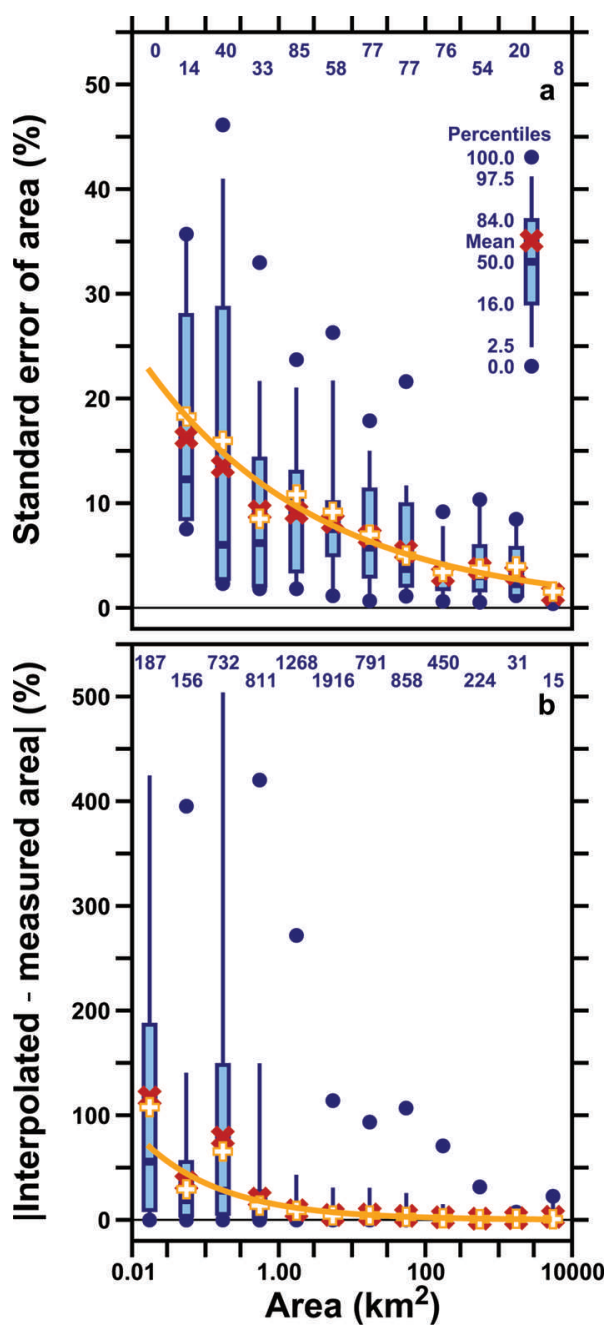

Fig. 2. (a) Uncertainty in glacier area. Measured areas, numbers of which are given at top, are grouped into logarithmic half-integer classes, and the distributions of their reported errors are shown as box-and-whisker plots. Orange curve (Eqn (2)) is fitted to 68th percentiles of area classes (open crosses). (b) Errors of interpolation in time; vertical scale is ten times that of (a). Orange curve (Eqn (4)) is fitted to 68th percentiles of area classes (open crosses).

to the 68th percentiles of the interpolated areas:

$$
e_{t}=e_{t 1} \widehat{S}^{\tau}
$$

The best-fitting parameters, for $e_{t}$ (expressed in $\% \mathrm{a}^{-1}$ ), were

\begin{tabular}{|c|c|c|c|c|c|c|c|c|c|c|c|c|}
\hline \multirow[b]{2}{*}{ Final pentad } & \multirow[b]{2}{*}{5} & \multirow[b]{2}{*}{10} & \multirow[b]{2}{*}{15} & \multirow[b]{2}{*}{20} & \multicolumn{3}{|c|}{ Years duration } & \multirow[b]{2}{*}{40} & \multirow[b]{2}{*}{45} & \multirow[b]{2}{*}{50} & \multirow[b]{2}{*}{$>50$} & \multirow[b]{2}{*}{ Mean } \\
\hline & & & & & 25 & 30 & 35 & & & & & \\
\hline 1960-65 & 0 & 4 & 0 & 0 & 0 & 0 & 0 & 0 & 0 & 1 & 1 & 24.2 \\
\hline 1965-70 & 0 & 0 & 3 & 0 & 0 & 2 & 0 & 0 & 0 & 0 & 0 & 21.0 \\
\hline 1970-75 & 0 & 13 & 1 & 26 & 0 & 0 & 0 & 0 & 0 & 0 & 3 & 19.3 \\
\hline $1975-80$ & 0 & 0 & 0 & 2 & 1 & 0 & 0 & 0 & 0 & 0 & 0 & 21.7 \\
\hline 1980-85 & 0 & 0 & 1 & 4 & 10 & 13 & 0 & 0 & 0 & 0 & 0 & 26.2 \\
\hline 1985-90 & 0 & 0 & 1 & 0 & 0 & 2 & 0 & 0 & 0 & 0 & 0 & 25.0 \\
\hline 1990-95 & 0 & 11 & 8 & 7 & 46 & 10 & 21 & 22 & 20 & 1 & 14 & 32.2 \\
\hline 1995-2000 & 1 & 6 & 0 & 2 & 34 & 17 & 6 & 22 & 10 & 38 & 33 & 39.8 \\
\hline 2000-05 & 15 & 5 & 26 & 1 & 3 & 46 & 26 & 17 & 15 & 25 & 20 & 33.2 \\
\hline 2005-10 & 0 & 2 & 0 & 0 & 0 & 0 & 0 & 7 & 0 & 0 & 0 & 33.3 \\
\hline
\end{tabular}

Table 1. Distribution of the duration of interpolated pentadal time series of measured glacier area and shrinkage rate, arranged by final pentad 


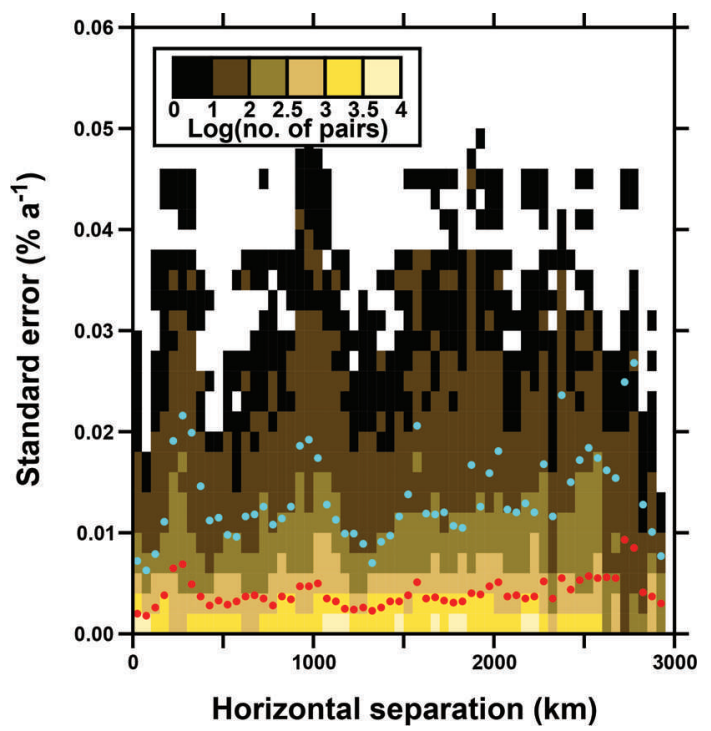

Fig. 3. Standard error (Eqn (5)) of the average shrinkage rates of pairs of glaciers and small glacierized regions, plotted as a function of the distance separating the members of each pair. Red and cyan dots: averages and 95th percentiles respectively of the shrinkagerate errors grouped into $50 \mathrm{~km}$ wide separation classes.

$e_{t 1}=14.3$ and $\tau=-0.379$. Eqn (4) was used to calculate errors for all interpolated areas, except that $e_{t}$ was set to zero for those at dates within 0.5 year of an area measurement.

The logarithms of $e_{s}$ and $e_{t}$ are perfectly correlated, but they summarize samples of single-object errors that are highly variable. We assume that on an object-by-object basis the measurement and interpolation errors are independent and can be added in quadrature, as in the rightmost column of Table 2, to obtain the total error in interpolated area estimates. Below $1 \mathrm{~km}^{2}$, interpolation is the dominant source of uncertainty. It doubles the total error in pentadal area estimates at $S \sim 0.25 \mathrm{~km}^{2}$, and at and below that size it is so large as to make interpolation unprofitable. From Eqns (2) and (4), the two error sources are equal at $S \sim 4 \mathrm{~km}^{2}$, so that interpolation increases the error by $\sim 40 \%$. At larger sizes interpolation makes a progressively smaller contribution. On regional scales, it is of course necessary to consider these size-dependent errors in the context of the distribution of glacier sizes. In most glacierized regions there are more small glaciers than large ones, but their large percentage errors will contribute only moderately to the absolute error in the glacierized area of the region.

Uncertainty in measured shrinkage rates (Eqn (1)) follows straightforwardly from the propagation of errors. The uncertainty standard error $\operatorname{se}(\Delta t)$ in the time span was assumed to be equal to one-quarter of the tabulated date range. Each interpolated pentadal rate is assigned the uncertainty of the measured rate by which it is bracketed.

\subsection{SPATIAL UNCERTAINTY}

Interpolation in space, drawing on correlated information from nearby measurements, is a possible remedy for filling unmeasured spatial gaps. Series of pentadal shrinkage rates were assembled from single glaciers and from glacierized regions extending over no more than two $0.5^{\circ}$ cells. Multiple series from the same object were averaged. Each series was then compared with all other series. As a measure
Table 2. Estimated errors (\% of area) of area measurement and area interpolation to pentadal dates

\begin{tabular}{lccc}
\hline Size & $e_{s}(\operatorname{Eqn}(2))$ & $e_{t}(\operatorname{Eqn}(4))$ & Total $\left[e_{s}^{2}+e_{t}^{2}\right]^{1 / 2}$ \\
$\mathrm{~km}^{2}$ & & & \\
& & & \\
\hline 0.01 & 25.1 & 81.9 & 85.6 \\
0.1 & 16.4 & 34.2 & 37.9 \\
1 & 10.7 & 14.3 & 17.9 \\
10 & 7.0 & 6.0 & 9.2 \\
100 & 4.6 & 2.5 & 5.2 \\
1000 & 3.0 & 1.0 & 3.2 \\
\hline
\end{tabular}

of spatial correlation, the quantity

$$
\delta \dot{S}_{d}=\left|\dot{S}_{A}(t)-\dot{S}_{B}(t)\right| / 2
$$

was calculated. This is the standard error of the average of the shrinkage rates of two objects $A$ and $B$ over pentad $t$, the objects being separated by distance $d(\mathrm{~km})$. Each pair of objects yielded up to ten pentadal errors over 1960-2010, and the total sample size was $\sim 252000$. The errors were grouped into cells (Fig. 3 ).

The simplest interpretation of Figure 3 is that there is no useful spatial coherence of shrinkage rates. As shown by the dots representing the 95th percentile, the uncertainty of any one rate as a predictor of a distant rate varies erratically, but it implies an additional $2 \sigma$ error of the order of $0.01 \% \mathrm{a}^{-1}$ no matter how far apart the two objects are. To rephrase this finding, gaps might as well be filled with the region-wide average as with any estimate based on nearby measurements. The extra uncertainty might be acceptable, because typical measured rates are commonly an order of magnitude greater, but there is an alternative to spatial interpolation.

The alternative to filling empty cells with the region-wide average shrinkage rate is to consider the dependence of shrinkage rate on initial glacier size $S_{0}$. This dependence is discernible more often than not at the scale of regions studied in the literature (Fig. 4), and for estimating regionwide average shrinkage rates it seems worthwhile to try to take it into account. The expression

$$
\dot{S}\left(S_{0}\right)=a S_{0}^{b}
$$

with $a=-0.36$ and $b=-0.34$ is used below to represent the impact of size dependence on estimates of shrinkage rate.

\section{RESULTS AND DISCUSSION}

Shrinkage-rate errors are substantial (Fig. 5). Of 3732 pentadal rates between 1960 and 2010, 65\% are indistinguishable from zero at the $2 \sigma$ level ( $41 \%$ at the $1 \sigma$ level). Just under one-third are more negative than 2 standard errors. Positive rates, representing glacier expansion, account for $6 \%$ of the sample, with $2 \%$ significantly positive. It seems reasonable, however, to adopt the working hypothesis that most glaciers are indeed shrinking, and that the preponderance of insignificant changes in the present dataset is due to lack of accuracy in measurements, many of which were obtained from topographic maps or by remote sensing with sensors of low resolution (such as the Landsat multispectral scanner). Considerable averaging of these measurements is therefore needed in order to increase statistical confidence. This is a subject for future investigation, because the number 


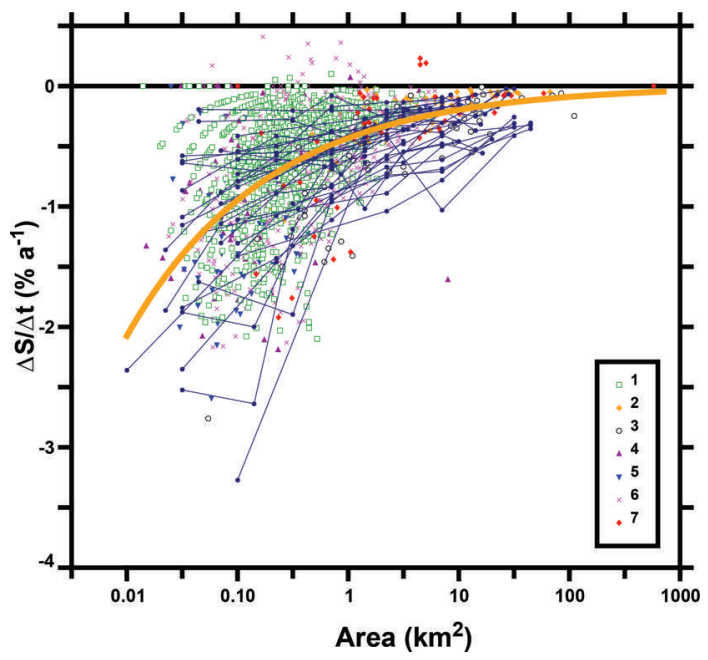

Fig. 4. Shrinkage rates as a function of initial glacier size from published graphs. 1. Hei basin, Qilian Shan (Wang and others, 2011); 2. upper Bhagirathi/Alaknanda basins, Garhwal Himalaya (Bhambri and others, 2011); 3. Tamor/Tista basins, Nepal and Sikkim (Racoviteanu and others, 2015); 4, 5. Bogda Shan south and north flanks, eastern Tien Shan (Li and others, 2011); 6. Ebi Nur basin, Tien Shan (Wang and others, 2014); 7.52 rates covering 1970-2005 for single glaciers from the main dataset. Connected blue dots: average rates within size-distribution classes (assigned unit weight; glacier numbers within classes are not always known). Orange curve: power law best fitting the negative members of the ensemble of shrinkage rates (Eqn (6)).

of degrees of freedom in the sample of rates is unknown and therefore so is the benefit that accrues from averaging. Another task for the future is the obvious one of continuing to seek ways of making more accurate measurements, but a third task is to revisit the error models of Section 3 to investigate whether they are too pessimistic. One practical step that would reduce the errors would be to report measurement dates more exactly.

In this regard it is instructive to consider the quantity

$$
\left(\frac{\operatorname{se}(\dot{S})}{\dot{S}}\right)^{2}=\left(\frac{\operatorname{se}(\Delta S)}{\Delta S}\right)^{2}+\left(\frac{\operatorname{se}(\Delta t)}{\Delta t}\right)^{2}
$$

Equation (7) says that the reciprocal of the quantity on the horizontal axis of Figure 5 is the root sum square of the fractional errors of the area change and the time span. In other words, rates are uncertain in proportion equally to the uncertainties of their numerators and their denominators. The treatment here of incomplete reporting of dates is to assign date ranges, such that a date of the form $Y Y Y Y$ has $\operatorname{se}(\Delta t)=0.25$ year and in general a date range has $\operatorname{se}(\Delta t)$ equal to one-quarter of the range. In many published reports, only date ranges are given for collections of areachange measurements for which exact dates are known by their authors for each of many glaciers. Out of conservatism, the date range divided by four is adopted here for each of these glaciers, but given fuller reporting a more exact date error, often equal to zero, could be assigned to each member of the collection.

We turn now to the geography and history of shrinkage. Measurements were judged to be too few before 1960 to warrant detailed analysis, but from 1960 to 2000 there is a steady improvement in coverage (Fig. 6). Nevertheless there are long gaps and some places where area change has never

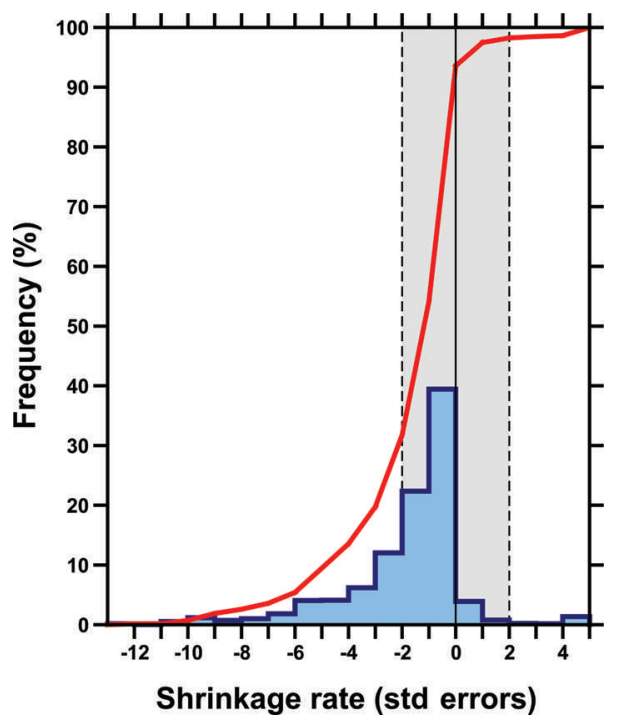

Fig. 5. Frequency distribution (blue histogram) and cumulative frequency distribution (red line) of errors in fractional shrinkage rate (Eqn (1)) derived from interpolated pentadal areas (such that measured rates are weighted by their time spans). Rates are divided by their standard errors. Grey rectangle: region within \pm 2 standard errors of zero.

been measured. The least well-known regions are the Pamir after 1980, the Hindu Kush, northern Pakistan, before 2000, and the Hengduan Shan area (southeastern Tibet, Sichuan and Yunnan) intermittently. Persistent cloud cover explains the intermittent coverage of the latter area, but where satellite imagery is available the poor coverage of other regions may be more plausibly explained by lack of attention from an overworked and under-supported remote-sensing community. It is unfortunate, however, that the poorly covered regions include some of the largest glaciers in High Mountain Asia.

Pentadal rates of glacier expansion exceeding $+0.1 \% \mathrm{a}^{-1}$ are rare (Fig. 7), and some of them no doubt represent surges and are thus, at best, indirect indicators of climatic forcing. Rates close to zero are common but not dominant. Shrinkage rates more negative than about $-0.8 \% \mathrm{a}^{-1}$ are widespread. The unweighted average of the rates in Figure 7 over all pentads is $-0.57 \% \mathrm{a}^{-1}$, but this generalization neglects many details that require fuller information. One of the possibly influential factors that cannot yet be quantified at the region-wide scale is the effect on shrinkage rates of debris cover and the related stagnation of flow (Scherler and others, 2011). Another is the acceleration of frontal ablation and retreat when proglacial lakes form and grow (Basnett and others, 2013). The most important factor that confounds interpretation of maps such as those of Figure 7 may be that they do not contain information about the variation of glacier size between gridcells.

Negligible and moderate shrinkage rates (light-blue and yellow cells in Fig. 7) form a clear spatial pattern that is a function of latitude or continentality, or possibly of both. Slow rates prevail from the southern Pamir and the Hindu Kush eastwards to the Karakoram and western Kun Lun, and onward to the eastern Kun Lun and the northern Tibetan Plateau. In the Tien Shan and Altai to the north, and the southern Tibetan Plateau and the Himalaya to the south, more rapid shrinkage is the rule. Moderate shrinkage rates 

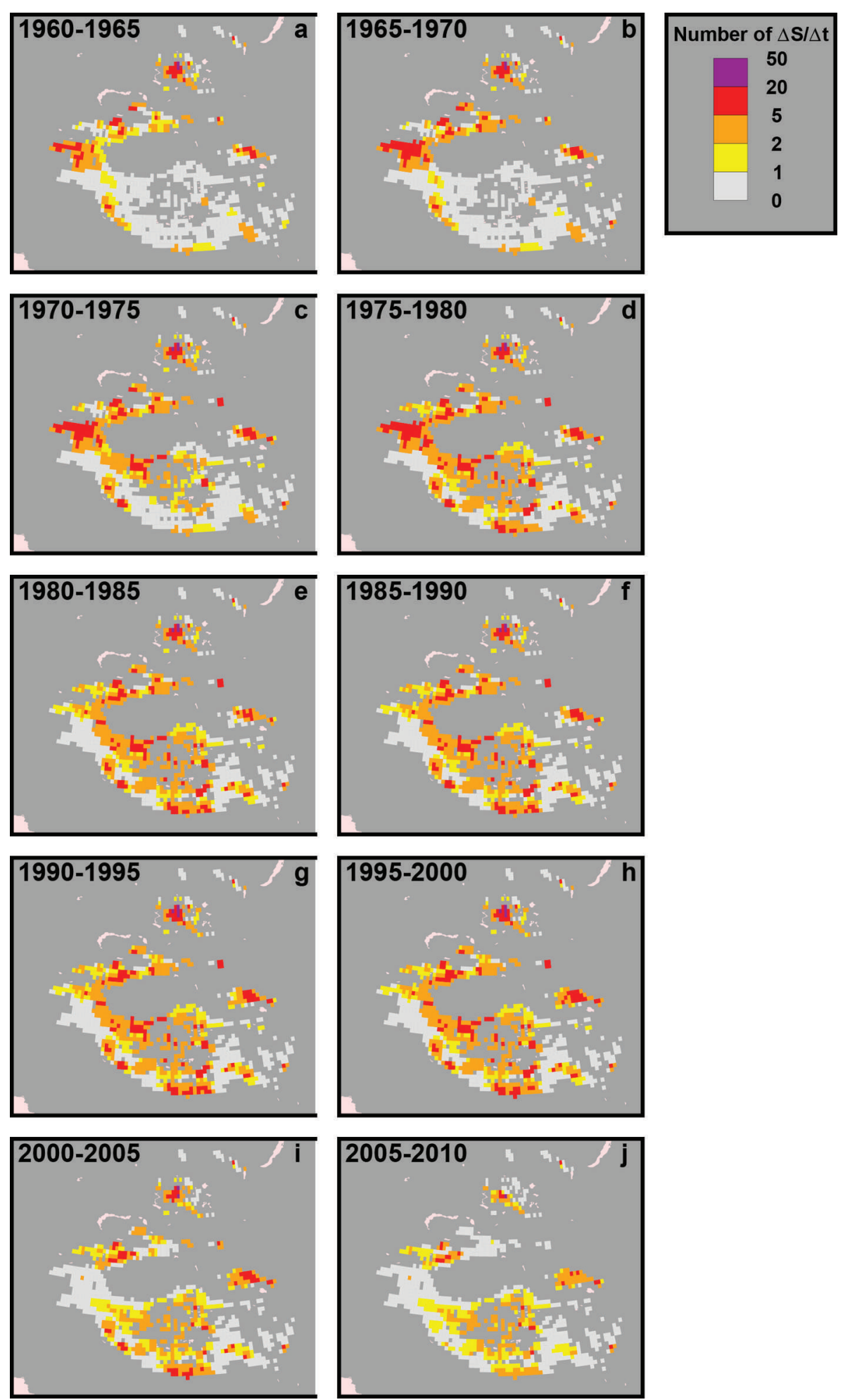

Fig. 6. Number of shrinkage rates per $0.5^{\circ}$ cell. These are interpolated pentadal rates, not original measurements.

also appear in the sparsely covered Hengduan Shan and the southeast.

Figure 8 summarizes the history of shrinkage rates as a function of latitude. Its time axis, however, must be viewed cautiously. The mean duration of the measurements is some decades (Table 1), and much of the temporal structure in the figure may be due to overlap between measurements. The real temporal resolution of the area-change dataset needs to be explored in more detail. Nevertheless, some zones exhibit notable persistence. Shrinkage is persistently rapid at $47-49^{\circ}$ and persistently slow at $34-37^{\circ}$. The latter zone contains the Karakoram. We saw in Figure 7 that in terms of shrinkage rates the 'Karakoram' anomaly (Hewitt, 2005) is a zonal feature extending well to the east of the Karakoram proper, in agreement with the mass-balance estimates of Kääb and others (2015). We now see in Figure 8 that it is also a long-lived feature.

Although climatic forcing (e.g. Janes and Bush, 2012) is beyond the present scope, and Figure 8 does not address the possible role of continentality in shaping the Karakoram anomaly, and perhaps in any case no simple zonal pattern should be expected, it seems clear that the anomaly is a 

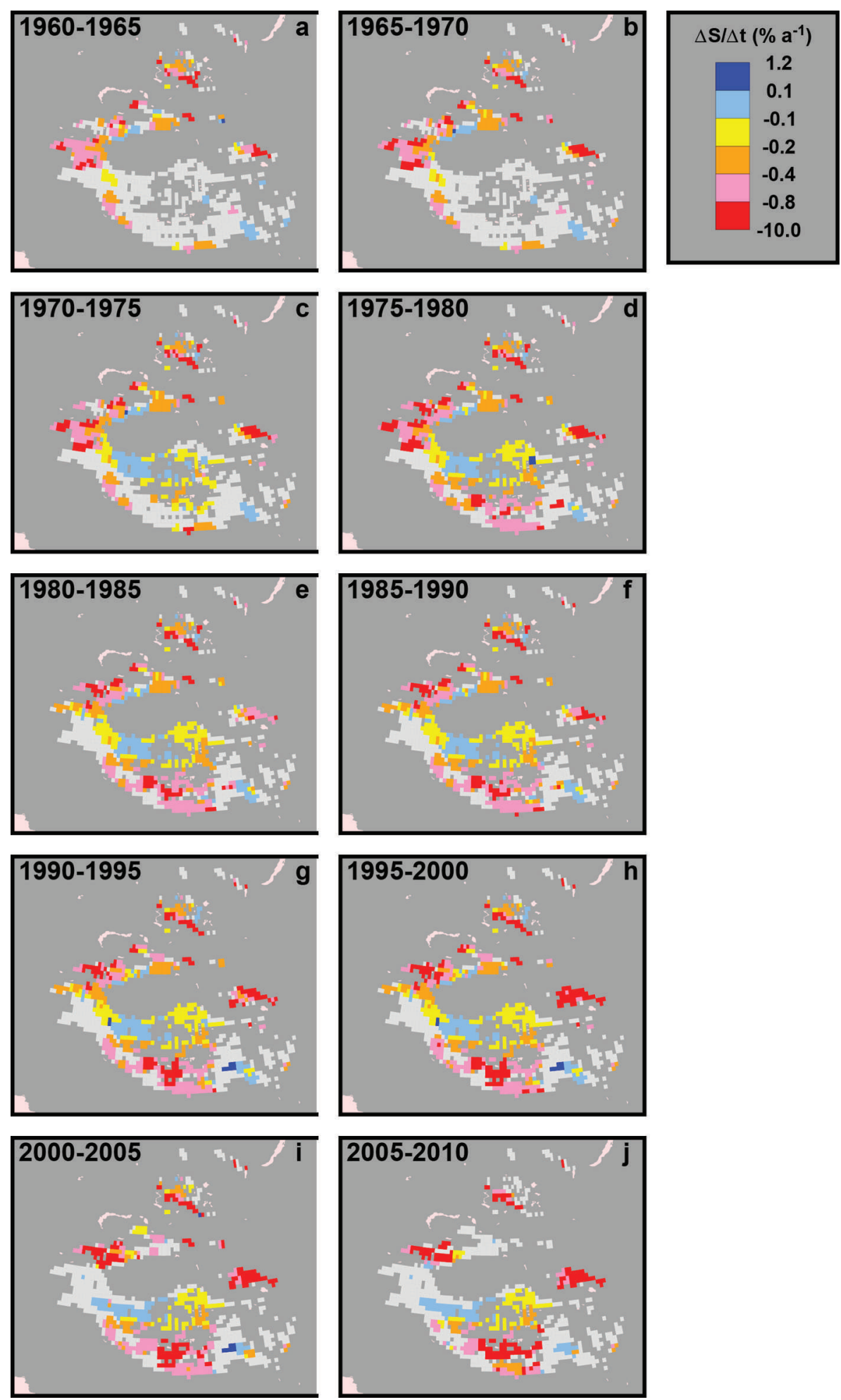

Fig. 7. Pentadal glacier shrinkage rates.

larger and more complex phenomenon than was originally believed. One factor that probably contributes to it is the size dependence of glacier shrinkage rate (Fig. 4). Glaciers at $34-37^{\circ}$ are larger, on average, than those at other latitudes (Fig. 8, right panel), and should be expected to shrink more slowly for that reason alone. In short, identifying and separating the factors that explain the Karakoram anomaly will be a large project.

Unweighted average shrinkage rates for all of High Mountain Asia are plotted with nominal error bars in Figure 9. When the rates are weighted by the glacierized areas of their cells (from Fig. 1b), shrinkage appears markedly slower, with the multi-decadal average shrinkage being reduced to $-0.34 \% \mathrm{a}^{-1}$. Cells with considerable ice cover contain glaciers that are shrinking more slowly. For example, cells exhibiting expansion $(\dot{S}>0)$ have an average ice cover of $18.7 \%$, against only $6.0 \%$ for cells exhibiting shrinkage.

The weighting also eliminates the acceleration of shrinkage since the 1990s that is seen in the unweighted averages. This may be understood in terms of greater relative representation of slow shrinkage during the 2000s. For example, gridcells exhibiting expansion account for $1.5 \%$ of 


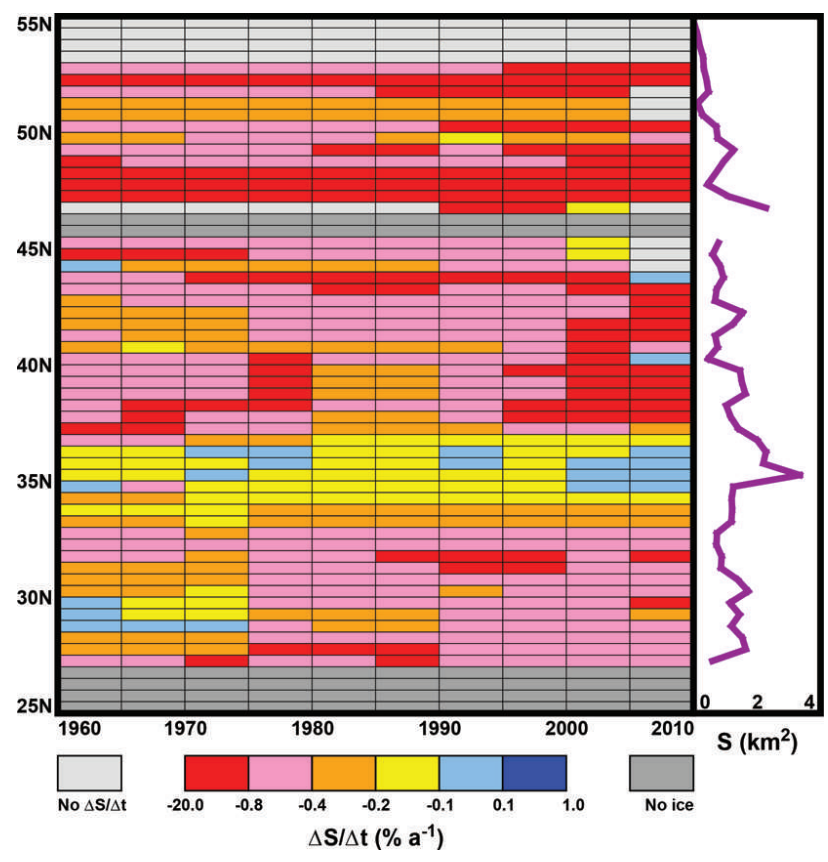

Fig. 8. Zonally averaged pentadal shrinkage rates. Panel at right shows zonal-mean glacier size, obtained from Figure $1 \mathrm{~b}$.

the sample before 2000 but for $6 \%$ of the post-2000 sample. The acceleration itself is due to greater relative representation of rapid shrinkage; pentadal rates more negative than $-0.6 \% \mathrm{a}^{-1}$ account for $27.5 \%$ of all rates before 2000 and $44.5 \%$ after. That is, the distribution of shrinkage rates is more dispersed during the 2000s. That cells with slow shrinkage or expansion have greater ice cover means that weighting by area has a very strong impact, and also that in region-wide terms the acceleration may be an artefact of sampling. These illustrative results call for further investigation, but are consistent with the documented tendency for shrinkage rate to depend on glacier size (Fig. 4).

The unweighted and area-weighted estimates in Figure 9 assume that the average of the interpolated measurements is a reliable predictor of the region-wide average rate. The stepped line in the figure is derived from the weighted averages by gap-filling with estimates based on the size dependence of shrinkage rates. Glaciers in the Randolph Glacier Inventory are assigned to the appropriate gridcell, and at cells without measurements the Randolph glacier areas (which are unchanging) are used to calculate the sum of rates implied by Eqn (6). The gap-filled multi-decadal estimate is $-0.40 \% \mathrm{a}^{-1}$, slightly faster than the corresponding area-weighted estimate. This is consistent with the unmeasured cells containing smaller glaciers than the measured cells: on average over 1960-2010, the mean glacier size in unmeasured cells is $1.18 \mathrm{~km}^{2}$, while in measured cells it is $1.53 \mathrm{~km}^{2}$.

\section{CONCLUSION}

This compilation of glacier shrinkage rates in High Mountain Asia will have value for placing local measurements in a wider spatial context, but it will also be valuable in still wider contexts. For example, for lack of a spatially resolved dataset of area changes the global-average glacier mass balance must currently be estimated based on unchanging glacier areas. Dyurgerov (2010) attempted to allow for

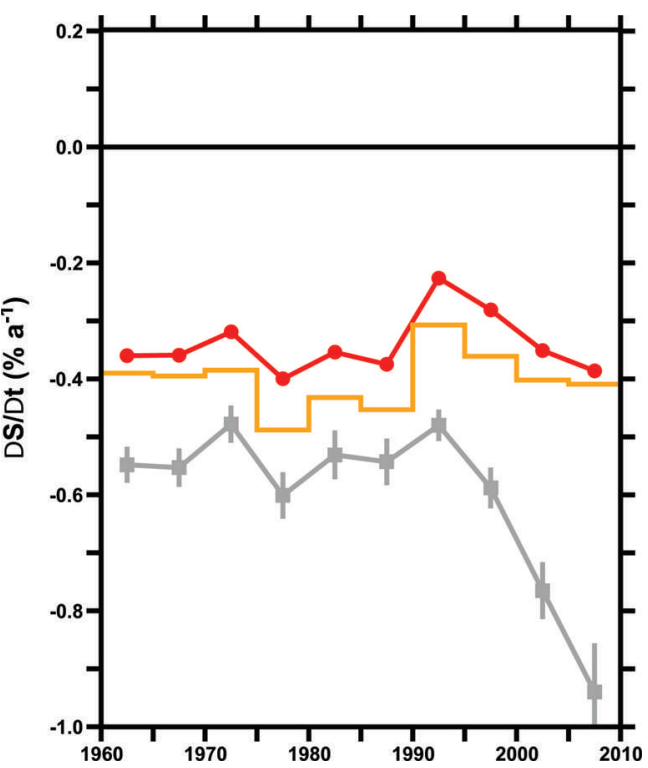

Fig. 9. Region-wide average shrinkage rates for 1960-2010. Squares with \pm 1 se error bars: unweighted averages of rates from glacierized cells (between 334 and 630 per pentad) to which measurements have been interpolated. Circles: average rates weighted by the glacierized area of each cell. Stepped line: areaweighted averages of cells with measurements, augmented by estimates from Eqn (6) (Fig. 4) for cells without measurements.

glacier shrinkage, but his sample of area changes was small compared with the present sample. Another broad context in which this compilation will prove valuable is the communication of facts about glacier changes to the public. Shrinkage is easier for non-specialists to grasp than are changes of volume and mass, which need to be explained with care if their implications are to be understood accurately.

Most area-change measurements cover $>5$ years, and most regional measurements appear in multiple $0.5^{\circ}$ cells. Even when areas are measured frequently, most methods are not accurate enough to resolve changes of shrinkage rate at the pentadal timescale. Many studies of glacier shrinkage in recent decades document faster shrinkage in the later than in the earlier measurements of time series, but this evidence has not survived interpolation and weighting by glacier size. Thus the maps presented above probably exaggerate the amount of realistic detail, although they have the advantage of showing, in condensed form, all the information available in the published literature.

Increasing the extent of coverage is an obvious goal. Studies achieving extensive coverage include Konovalov and Shchetinnicov (1994), Liu and others (2006) and Konovalov and Desinov (2007), but they relied in part on sampling regional subsets of glaciers. Continuing technical advances, as illustrated by Wei and others (2014), can be expected to make extensive coverage more feasible, and Figure 6 may assist in the targeting of poorly studied regions.

The methodology proposed here has potential for more efficient treatment of multiple measurements of glacier area change at both broader (global) and narrower (local) scales. In detail, resolvable difficulties are likely to arise, but the essential ideas are:

Replicate measurements of the same or overlapping regions or time spans should be accepted unless 
obviously flawed, and should be seen as opportunities to gauge uncertainties 'from the outside'.

Interpolation to fixed dates is an exercise with quantifiable errors, as shown here. Notwithstanding the risks of interpolating the areas of very small glaciers, the increment of uncertainty may be acceptable in many contexts, especially when the gain in comparability between independent studies is taken into account.

Interpolation to a fixed spatial grid breaks the connection to individual glaciers, and may or may not be advantageous. Considering the extremely strong variability of single-glacier behaviour, it may as a minimum be useful for purposes of graphical display, and in some contexts for reducing the amount of computation.

Of the factors that affect shrinkage rates, the initial size of the glacier is both known and strongly influential, so it can and should be corrected for in comparative studies. Equation (6) is a possible prototype for a correction procedure. Its scaling factor a can be understood as the shrinkage rate of a notional glacier with an area of $1 \mathrm{~km}^{2}$, and it is plausible to speculate that inter-regional differences in a will contain a relatively pure climatic signal. More of the multisets that are needed for estimating it, and for exploring the information content of variations in the exponent $b$, would therefore be valuable.

Further to the last of these ideas, the greater variability of the shrinkage rate of smaller glaciers (Fig. 4) deserves closer attention. It arises from (1) greater random measurement uncertainty at smaller sizes (Fig. 2a), (2) the geometrical fact that smaller glaciers tend to have greater ratios of area to margin length, the margin being where the shrinkage happens, and (3) doubtless a contribution from the greater impact, other things being equal, of climatic forcing on glaciers with shorter response times. Item (1) is a source of spurious variability. Item (2) makes it hazardous to compare rates between glaciers of different size, but is also entangled with questions such as whether some smaller glaciers shrink faster because, on average, they are at lower elevations, and whether some others do not because they are nourished by avalanching. Item (3) is demonstrably real (e.g. from the viewpoint of Fig. 2b), but with current knowledge it is not practical to isolate the size-dependent climatic and topographic signals from the less interesting item (1) and the purely geometric aspects of item (2).

\section{ACKNOWLEDGEMENTS}

I thank Adina Racoviteanu for a thorough and thoughtful review, and several hundred colleagues for labours without which this study would have been impossible.

\section{REFERENCES}

Arendt AA and 77 others (2014) Randolph Glacier Inventory: a dataset of global glacier outlines: version 4.0. (GLIMS Technical Report) National Snow and Ice Data Center, Boulder, CO. Digital media

Basnett S, Kulkarni AV and Bolch T (2013) The influence of debris cover and glacial lakes on the recession of glaciers in Sikkim
Himalaya, India. J. Glaciol., 59(218), 1035-1046 (doi: 10.3189/ 2013/JoG12JoG12J184)

Bhambri R, Bolch T, Chaujar RK and Kulshreshtha SC (2011) Glacier changes in the Garhwal Himalaya, India, from 1968 to 2006 based on remote sensing. J. Glaciol., 57(203), 543-556 (doi: 10.3189/002214311796905604)

Dyurgerov MB (2010) Reanalysis of glacier changes: from the IGY to the IPY, 1960-2008. Mater. Glyatsiol Issled., 110, 1-116

Hastings DA and Dunbar PK eds (1999) Global Land One-kilometer Base Elevation (GLOBE). (Key to Geophysical Records Documentation No. 34) Digital Elevation Model, Version 1.0. National Geophysical Data Center, National Oceanic and Atmospheric Administration, Boulder, CO

Hewitt K (2005) The Karakoram anomaly? Glacier expansion and the 'elevation effect'. Mt. Res. Dev., 25(4), 332-340 (doi: 10.1659/0276-4741(2005)025[0332:TKAGEA]2.0.CO;2)

Janes TJ and Bush ABG (2012) The role of atmospheric dynamics and climate change on the possible fate of glaciers in the Karakoram. J. Climate, 25(23), 8308-8327 (doi: 10.1175/JCLID-11-00436.1)

Kääb A, Nuth C, Treichler D and Berthier E (2015) Contending estimates of early 21 st century glacier mass balance over the Pamir-Karakoram-Himalaya. Cryosphere, 9, 557-564 (doi: 10.5194/tc-9-557-2015)

Konovalov V and Desinov L (2007) Remote sensing monitoring of the long-term regime of the Pamirs glaciers. IAHS Publ. 316 (Symposium at Perugia 2007 - Vulnerability of Societies to Water Related Risks), 149-156

Konovalov VG and Shchetinnicov AS (1994) Evolution of glaciation in the Pamiro-Alai mountains and its effect on river run-off. J. Glaciol., 40(134), 149-157

Li KM, Li ZQ, Gao WY and Wang L (2011) Recent glacial retreat and its effect on water resources in eastern Xinjiang. Chinese Sci. Bull., 56(33), 3596-3604 (doi: 10.1007/s11434011-4720-8)

Liu SY and 6 others (2006) Glacier retreat as a result of climate warming and increased precipitation in the Tarim river basin, northwest China. Ann. Glaciol., 43, 91-96

Pfeffer WT and 75 others (2014) The Randolph Glacier Inventory: a globally complete inventory of glaciers. J. Glaciol., 60(221), 537-552 (doi: 10.3189/2014JoG13J176)

Racoviteanu A, Arnaud Y, Williams M and Manley WF (2015) Spatial patterns in glacier area and elevation changes from 1962 to 2006 in the monsoon-influenced eastern Himalaya. Cryosphere, 9, 505-523 (doi: 10.5194/tc-9-505-2015)

Scherler D, Bookhagen B and Strecker MR (2011) Spatially variable response of Himalayan glaciers to climate change affected by debris cover. Nature Geosci., 4(3), 156-159 (doi: 10.1038/ ngeo1068)

Wang L, Li ZQ, Wang FT and Edwards R (2014) Glacier shrinkage in the Ebinur lake basin, Tien Shan, China, during the past 40 years. J. Glaciol., 60(220), 245-254 (doi: 10.3189/ 2014JoG13J023)

Wang PY, Li ZQ and Gao WY (2011) Rapid shrinking of glaciers in the middle Qilian Mountain region of northwest China during the last $\sim 50$ years, J. Earth Sci., 22(4), 539-548 (doi: 10.1007/ s12583-011-0195-4)

Wei JF and 6 others (2014) Surface-area changes of glaciers in the Tibetan Plateau interior area since the 1970s using recent Landsat images and historical maps. Ann. Glaciol., 55(66), 213-222 (doi: 10.3189/2014AoG66A038)

Zemp $M$ and 10 others (2014) Introduction: Global glacier monitoring - a long-term task integrating in situ observations and remote sensing. In Kargel JS, Leonard GJ, Bishop MP, Kääb A and Raup $\mathrm{BH}$ eds Global Land Ice Measurements from Space. Springer Praxis, Berlin, 1-21 (doi: 10.1007/978-3540-79818-7) 